\title{
Celulitis y erisipela: Manejo en atención primaria
}

\author{
ALBERTO FICA C. ${ }^{1}$
}

\section{Cellulitis and erysipelas: Management in primary care}

Cellulitis and erysipelas are important causes of morbidity and hospital admissions. Erysipelas has a characteristic clinical manifestation and is associated typically to Streptococcus pyogenes and less frequently to group B, C or G Streptococci. The most relevant risk factors for erysipelas are lymphoedema and local skin lesions. Raised anti-streptolysin $\mathrm{O}$ antibodies may support diagnosis of erysipelas but are present in no more than $40 \%$ of cases. Therapy of choice is penicillin or amoxicillin and the length and route of administration is dictated by clinical severity. Cellulitis is a spreading infection of skin and subcutaneous structures without palpable margins. It has a broader etiological spectrum than erysipelas including S. pyogenes and Staphylococcus aureus. Resistance of the latter microrganism to penicillin demands an antimicrobial scheme based either on cloxacillin, antistaphylococcal cefalosporins (i.e. cefazolin), clindamycin or beta lactam - beta-lactamase inhibitor combinations. Emergence of invasive $S$. pyogenes infections imposes a proper and early recognition of this condition. Clues for this suspicion are hypotension, a rapidly evolving condition, tisular necrosis and rush. These infections should be managed by parenteral antibiotics, supportive therapy and aggressive surgical debridement.

Key words: Erysipelas, Cellulitis, Treatment.

\section{Introducción}

Los cuadros de celulitis y erisipela representan una causa importante de morbilidad, hospitalización y en ocasiones, de mortalidad en la población general. Así como los cuadros de erisipela tienen una manifestación clínica, alteraciones patológicas y una etiología característica, los cuadros de celulitis tienen un mayor espectro etiológico y clínico e imponen mayores dificultades en el diagnóstico y tratamiento. No obstante, para ninguna de las dos condiciones se ha aclarado el valor diagnóstico predictor de los síntomas o signos asociados a ellas o un gold standard diagnóstico. El rendimiento de las pruebas microbiológicas es de baja sensibilidad en ambos casos y por ello, el diagnóstico se efectúa predominantemente en forma clínica. La emergencia en las últimas dos décadas de cuadros invasores asociados a Streptococcus pyogenes, algunos de ellos manifestados como cuadros de fascitis necro- sante, resalta la importancia del diagnóstico diferencial apropiado en estas condiciones.

Este artículo ha sido preparado para facilitar el reconocimiento clínico de estas enfermedades, sus factores de riesgo, las pruebas diagnósticas disponibles y las bases del tratamiento. Se entrega además, información sobre el manejo de los casos recurrentes y el reconocimiento apropiado de las enfermedades invasoras asociadas a $S$. pyogenes. No se revisa en esta publicación otras formas de infecciones cutáneas tales como el impétigo o las asociadas al pie diabético.

\section{Definiciones, diagnóstico clínico y causas}

Los cuadros de erisipela señalan una inflamación infecciosa aguda en placas que afecta la epidermis, asociada generalmente a fiebre, linfangitis, leucocitosis y ocasionalmente adenopatías regionales ${ }^{1,2}$. El compromiso superficial de la piel

\footnotetext{
Sección de Infectología. Hospital Clínico Universidad de Chile.
}

Recibido: 6 noviembre 2002

Aceptado: 11 abril 2003 
explica los límites precisos de esta placa, la que además se presenta con bordes solevantados (Tabla 1). El cuadro es de inicio agudo, con fiebre alta (sobre $38^{\circ} \mathrm{C}$ ) y calofríos, lo que motiva la consulta precoz. Una gran parte de los pacientes con erisipela en las extremidades inferiores presentará además, una puerta de entrada cutánea cercana. El diagnóstico se establece de acuerdo al conjunto de manifestaciones clínicas ya que no se ha definido el valor predictor real de cada uno de los síntomas y signos asociados, en parte debido a la ausencia de un gold standard diagnóstico. La erisipela es una infección aguda asociada a estreptococos, especialmente $S$. pyogenes y en forma menos frecuente por estreptococos $\beta$-hemolíticos de los grupos B, C $\mathrm{o}^{2,3}$. Debido a que los cuadros de erisipela no se asocian en general a estreptococos resistentes a diferentes antimicrobianos, esta afección puede ser universalmente tratada con penicilina o derivados. Este mismo fenómeno explica también la baja presión clínica para contar con un método de confirmación microbiológica.

A diferencia de los cuadros de erisipela, la celulitis denota una inflamación más profunda y no necesariamente de causa infecciosa. No tiene un correlato anátomo patológico preciso y no presenta una placa solevantada ni bordes definidos. Puede presentarse con fiebre, compromiso del estado general, bacteriemia y/o abscesos locales. A pesar de que una gran parte de estos cuadros se asocia a $S$. pyogenes (y ocasionalmente a Streptococcus agalactiae) ${ }^{3}$, también participa como causa etiológica Staphylococcus aureus, lo que dificulta las opciones terapéuticas por la resistencia casi universal que esta especie presenta ante penicilina en la comunidad.
De las causas no infecciosas que aparentan una celulitis, la tromboflebitis superficial debe ser destacada por su importancia en atención primaria. En esta última condición es posible en ocasiones palpar un trayecto venoso superficial inflamado, el paciente habitualmente no tiene fiebre alta y responde favorablemente al uso de antiinflamatorios no esteroidales, sin necesidad de utilizar antimicrobianos.

\section{Diagnóstico etiológico}

El diagnóstico microbiológico de estas condiciones es de bajo rendimiento global y puede ser intentado mediante hemocultivos (bajo $5 \%$ de positividad), infiltraciones locales con solución salina al $9 \%$ estéril y cultivo del aspirado, o mediante cultivo del contenido de lesiones bulosas indemnes ${ }^{4}$. En aproximadamente $40 \%$ de los pacientes afectados por erisipela se observa un aumento en los títulos de antiestreptolisina $\mathrm{O}$ (ASO) sobre los valores establecidos como normales en el ámbito local ${ }^{4}$. La importancia de este hallazgo radica en que permite establecer una etiología estreptocóccica para el cuadro observado, facilitando la decisión terapéutica. El diagnóstico también se puede efectuar en muestras de biopsia cutáneas tomadas con propósitos de investigación, mediante pruebas de aglutinación o IFD contra diferentes antígenos estreptocó$\operatorname{ccicos}^{3,5}$ (Tabla 2).

A pesar del bajo rendimiento de las técnicas disponibles, ellas deben ser solicitadas en los pacientes que se hospitalizan. Esta solicitud cobra importancia al considerar la reemergencia de las infecciones invasoras por S. pyogenes (la

\section{Tabla 1.Criterios diagnósticos tradicionales en el reconocimiento de erisipela y celulitis}

\section{Erisipela}

- Inflamación cutánea bien demarcada y solevantada.

- Inicio agudo (<24 horas), asociada a fiebre $\left(>38^{\circ} \mathrm{C}\right)$ o calofríos.

- Lesión generalmente unilateral $(98 \%)$ que afecta predominantemente la pierna o el pie (85\%).

- Factores de riesgo presentes en gran parte de los pacientes (linfedema, puerta de entrada local, etc)*.

- Un bajo porcentaje de los pacientes puede presentar títulos de anticuerpos antiestreptolisina O (ASO) positivos al inicio o durante el seguimiento serológico $(\sim 40 \%)$.

\section{Celulitis}

- Inflamación cutánea de límites imprecisos y no solevantada.

- Inicio agudo y asociado a fiebre en ocasiones.

- Factores de riesgo presentes similares a los de erisipela, a los que se agrega safenectomía reciente.

* ver detalles en Tabla 3. 
Tabla 2. Estrategias diagnósticas microbiológicas y serológicas disponibles en casos de celulitis o erisipela

\section{Métodos microbiológicos}

- Cultivo del contenido de lesiones bulosas (<5\% de los casos).

- Cultivo de aspirado de infiltración de solución salina al $9 \%$ estéril en la lesión.

- Hemocultivos (<5\% positividad, excepto en cuadros invasores por S. pyogenes).

- Inmunofluorescencia directa o aglutinación con partículas de látex para diferentes antígenos estreptocóccicos en muestras de biopsia cutánea (en casos de investigación).

\section{Métodos serológicos}

- Títulos elevados de anticuerpos antiestreptolisina O (ASO) al inicio o durante el seguimiento (40\% positividad, asociados a $S$. pyogenes o estreptococos del grupo C o G).

- Detección de anticuerpos anti-hialuronidasa o anti DNAasa B (S. pyogenes, estreptococos grupo C o G).

- Detección de anticuerpos anti- $\alpha$ lisina o anti-nucleasa de $S$. aureus.

mayor parte de las veces asociadas a hemocultivos positivos), la necesidad de conocer la susceptibilidad del agente a macrólidos en casos de alergia a $\beta$-lactámicos y, por la posibilidad de simplificar el tratamiento, antes o después del alta del paciente, en aquellos casos donde se logra documentar una celulitis de causa estreptocóccica (Figura 1).

\section{Factores de riesgo para erisipela y celulitis}

Estudios recientes han permitido precisar condiciones locales y sistémicas que predisponen al desarrollo de erisipela ${ }^{6}$. La importancia de reconocer estos factores es determinante para evitar

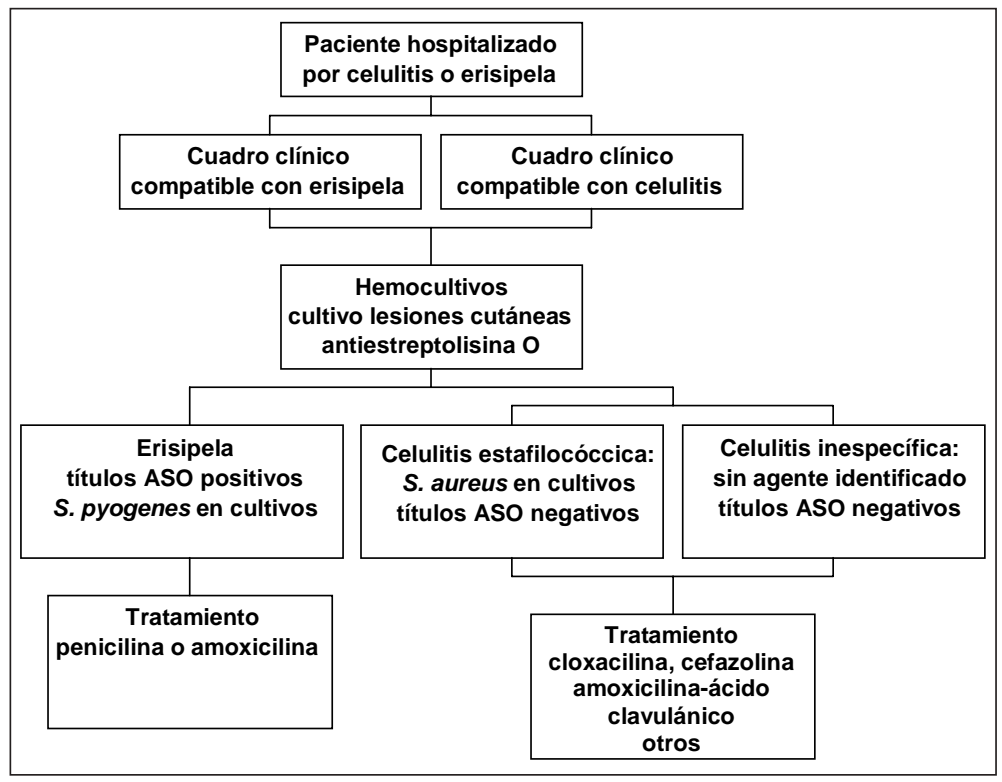

Figura 1. un primer episodio o la recurrencia de esta enfermedad. Los factores de riesgo más importantes son la presencia de linfedema y una alteración cutánea local. Otros factores corresponden a la presencia de insuficiencia venosa, edema de la extremidad, sobrepeso u obesidad. Estos estudios no han logrado demostrar que la diabetes mellitus sea un factor de riesgo independiente ${ }^{6}$. El factor más frecuente en la población es la presencia de intertrigo fisurado. Los factores de riesgo para celulitis se sobreponen a los de erisipela, a los que se agrega safenectomía o injertos venosos autólogos ${ }^{7-10}$.

\section{Bases terapéuticas}

Los objetivos del tratamiento de estos cuadros cutáneos son aliviar los síntomas del paciente, detectar y manejar oportunamente los casos con infecciones invasoras y evitar las recurrencias, mediante el manejo de los factores de riesgo. Para este propósito se debe considerar la hospitalización en pacientes con cuadros severos o en los que no se pueda asegurar un tratamiento adecuado en forma ambulatoria, prescripción de antimicrobianos, descartar diagnósticos alternativos si es necesario y manejar los factores de riesgo antes o después del alta (Tabla 3).

Se debe mencionar que a pesar de tratarse de afecciones frecuentes, existen escasos 
Tabla 3. Bases terapéuticas del manejo de pacientes con celulitis o erisipela

- Considerar hospitalización si el paciente presenta un cuadro severo o hay limitaciones para un adecuado manejo ambulatorio.

- Tratamiento con antimicrobianos de elección. Erisipela: penicilina G sódica parenteral o amoxicilina (clindamicina, eritromicina o cefalosporinas en casos de alergia a $\beta$-lactámicos). Celulitis: $\beta$-lactámico estable a $\beta$-lactamasas de $S$. aureus (cloxacilina o amoxicilina-ácido clavulánico) o cefalosporinas antiestafilocóccicas (cefazolina). En caso de alergia a $\beta$-lactámicos, utilizar cefazolina o clindamicina.

- Manejo de los factores de riesgo. Antifúngicos tópicos para manejo del intertrigo (clotrimazol, miconazol, ciclopirox $\mathrm{u}$ otro con cobertura sobre Candida albicans y en crema). Control obesidad, manejo insuficiencia venosa y/o edema.

estudios con diseño adecuado para el tratamiento de estos pacientes. Los aspectos que no han sido aclarados incluyen la dosis y vía óptima de administración de los antimicrobianos de elección y la duración del tratamiento. Empíricamente los pacientes con mayor compromiso sistémico son tratados inicialmente por vía parenteral y durante aproximadamente 14 a 21 días.

La asociación universal de los casos de erisipela con una etiología estreptocóccica permite plantear en estos casos, un tratamiento de elección con penicilinas o derivados cercanos. En contraste, la etiología en los cuadros de celulitis, no necesariamente está relacionada con estreptococos, sino que también con $S$. aureus, especie que es resistente a las penicilinas debido a la presencia de $\beta$-lactamasas. Por ello, el tratamiento de elección en casos de celulitis debe plantearse ya sea con penicilinas estables a estas enzimas (cloxacilina), combinaciones con inhibidores de $\beta$-lactamasas (amoxicilina-ácido clavulánico), cefalosporinas antiestafilocóccicas (cefazolina) o lincosamidas (clindamicina) (Tabla 3, Figura 1).

Aproximadamente 7 a 10\% de los aislamientos de $S$. pyogenes en la Región Metropolitana son resistentes a diferentes macrólidos ${ }^{11}$ y una fracción de los aislamientos de $S$. aureus de la comunidad es también resistente a estos compuestos.

\section{Selección de compuestos parenterales}

Los cuadros de erisipela o celulitis generan cierta confusión sobre el compuesto o combinación ideal para el tratamiento inicial, habitualmente se utiliza la vía parenteral. Las opciones parenterales más utilizadas se señalan en la Tabla 4.

En esta última tabla se puede apreciar que penicilina (o derivados cercanos como amoxicilina) tiene la mayor actividad antimicrobiana contra $S$. pyogenes y otros estreptococos (CIM $0,005 \mu \mathrm{g} / \mathrm{mL}$ para penicilina versus $0,12 \mu \mathrm{g} / \mathrm{mL}$ cefazolina y 0,04 a $2 \mu \mathrm{g} / \mathrm{mL}$ para cloxacilina) $)^{12,13}$.

Tabla 4. Actividad antibacteriana y parámetros farmacocinéticos de principales compuestos disponibles para el tratamiento de celulitis o erisipela

\begin{tabular}{|c|c|c|c|}
\hline Parámetro & Cefazolina & Cloxacilina & Penicilina G \\
\hline $\begin{array}{l}\text { Actividad contra } S \text {. pyogenes } \\
\text { y otros estreptococos*. }\end{array}$ & $0,12 \mu \mathrm{g} / \mathrm{ml}$ & $0,04-2 \mu \mathrm{g} / \mathrm{ml}$ & $0,005 \mu \mathrm{g} / \mathrm{ml}$ \\
\hline Actividad contra $S$. aureus $*$ & $0,5 \mu \mathrm{g} / \mathrm{ml}$ & $0,4-2 \mu \mathrm{g} / \mathrm{ml}$ & $25 \mu \mathrm{g} / \mathrm{ml}$ (resistente) \\
\hline Concentración plasmática máxima & $80 \mu \mathrm{g} / \mathrm{ml}$ & $70-100 \mu \mathrm{g} / \mathrm{ml}$ & $20 \mu \mathrm{g} / \mathrm{ml}$ \\
\hline Dosis y frecuencia & $1 \mathrm{~g} \mathrm{c} / 6-8 \mathrm{~h} \mathrm{ev}$ & $1-2 \mathrm{~g} \mathrm{c} / 4-6 \mathrm{~h} \mathrm{ev} \mathrm{u}$ oral & 1-6 millones $\mathrm{U} \mathrm{c} / 4 \mathrm{~h}$ ev \\
\hline $\begin{array}{l}\text { Frecuencia de flebitis durante } \\
\text { uso endovenoso }\end{array}$ & 1 a $5 \%$ & $13 \%$ & No disponible \\
\hline Costo aproximado** & 418 pesos por $\mathrm{g}$ & 602 pesos por $\mathrm{g}$ & 85 pesos por 1 millón $U$ \\
\hline Indicación & $\begin{array}{l}\text { Erisipela o celulitis } \\
\text { inespecífica }\end{array}$ & $\begin{array}{l}\text { Erisipela o celulitis } \\
\text { inespecífica }\end{array}$ & $\begin{array}{l}\text { Erisipela o celulitis } \\
\text { estreptocóccica }\end{array}$ \\
\hline
\end{tabular}

* CIM habitual o promedio; **Hospital Clínico U. de Chile año 2002. 
Se observa también, que tanto cefazolina (cefalosporina antiestafilocóccica) como cloxacilina, tienen una actividad equivalente ante $S$. aureus y no difieren en su actividad sobre los estreptococos; sus concentraciones plasmáticas máximas son además similares. Cefazolina y cloxacilina difieren en su potencial de provocar flebitis en el sitio de punción, siendo mayor en el caso de cloxacilina (13\% versus 1 a $5 \%$ para cefazolina $)^{14}$ y escasamente en la frecuencia de administración diaria ( 3 a 4 veces al día en el caso de cefazolina y 4 a 6 veces al día para cloxacilina). El costo de adquisición de ambos fármacos es levemente superior para cloxacilina (Tabla 4).

Las propiedades comentadas pueden inclinar la balanza hacia la selección de cloxacilina o cefazolina endovenosa en aquellos cuadros de celulitis donde no se ha aclarado su etiología estreptocóccica. Se debe recordar al lector que el uso combinado de penicilina y cloxacilina en casos de erisipela no se justifica, debido a la cobertura adecuada anti estreptocóccica lograda con penicilina. De la misma manera, el uso combinado de ambos fármacos tampoco se justifica en casos de celulitis, por la cobertura adecuada de cloxacilina contra estreptococos y S. aureus. En casos de sospecha de bacteriemia o bacteriemia documentada, se deben utilizar dosis máximas de penicilina o cloxacilina/cefazolina según si se trate de un caso de erisipela o celulitis, respectivamente.

Las penicilinas de depósito pueden ser utilizadas en el tratamiento de pacientes con erisipela, aunque no se dispone de ensayos clínicos controlados para este tipo de compuestos. En Chile, diversas formulaciones con clemizol están disponibles para este propósito, aunque su dosis y frecuencia de administración no ha sido definida para esta condición.

Amoxicilina es una opción terapéutica adecuada para el tratamiento oral en casos de erisipela o celulitis estreptocóccica sin mayor compromiso sistémico, o luego de un tratamiento parenteral inicial con penicilina. En caso de celulitis estafilocóccica, los pacientes pueden ser tratados con cloxacilina, amoxicilina-ácido clavulánico o clindamicina (Tabla 5). Lincomicina está relacionada con este último, pero tiene menos actividad bactericida, su absorción es interferida en mayor grado por los alimentos y logra una menor concentración plasmática en comparación con clindamicina ${ }^{15}$. Su costo de adquisición es además elevado.

A pesar de la prevalencia de ambas condiciones, se dispone de escasos estudios randomizados y controlados que hayan comparado las alternativas disponibles. En general los resultados son similares en eficacia y reacciones adversas, utilizando diferentes compuestos ${ }^{16-21}$. En contraste, los costos de adquisición son muy discrepantes, siendo los compuestos tradicionales cloxacilina o amoxicilina, de menor costo (Tabla 5).

La utilidad de los corticoesteroides no ha sido evaluada en el manejo de los cuadros de erisipela.

\section{Manejo de erisipela recurrente}

Los cuadros recurrentes deben ser tratados con los mismos esquemas que el cuadro inicial y además se debe identificar los factores de riesgo posiblemente involucrados. En aquellos casos donde la recurrencia no se puede controlar y ésta es frecuente, se puede indicar eritromicina

Tabla 5. Alternativas de tratamiento por vía oral

\begin{tabular}{lllll}
\hline Parámetro & Cloxacilina & Amoxicilina & $\begin{array}{l}\text { Amoxicilina/ } \\
\text { ácido clavulánico }\end{array}$ & Clindamicina* \\
\hline Indicaciones & $\begin{array}{l}\text { Erisipela o celulitis } \\
\text { inespecífica }\end{array}$ & $\begin{array}{l}\text { Erisipela o celulitis } \\
\text { estreptocóccica }\end{array}$ & $\begin{array}{l}\text { Erisipela o celulitis } \\
\text { inespecífica }\end{array}$ & $\begin{array}{l}\text { Erisipela o celulitis } \\
\text { inespecífica }\end{array}$ \\
Dosis y frecuencia & $500 \mathrm{mg} \mathrm{c} / 6 \mathrm{~h}$ & $500 \mathrm{mg} \mathrm{c} / 8 \mathrm{~h}$ & $\begin{array}{l}500 / 125 \mathrm{mg} \mathrm{c} / 8 \mathrm{~h} \\
875 / 125 \mathrm{mg} \mathrm{c} / 12 \mathrm{~h}\end{array}$ & $300 \mathrm{mg} \mathrm{c} / 6 \mathrm{~h}$ \\
$\begin{array}{l}\text { Efecto de las comidas } \\
\text { sobre la absorción }\end{array}$ & Disminuye absorción & Sin mayor efecto & Sin mayor efecto & Sin mayor efecto \\
Costo de adquisición** $\sim 2.400$ pesos & $\sim 1.900$ pesos & $\sim 17$ a 25 mil pesos & $\sim 43$ mil pesos \\
\hline
\end{tabular}

* cepas resistentes a clindamicina descritas en la comunidad para $S$. aureus y S. pyogenes. ** Según datos aparecidos en la revista Kayros $N^{\circ} 149$, junio del 2002; costo calculados para un tratamiento por 10 días sin incluir una terapia parenteral inicial. 
profiláctica en dosis de $250 \mathrm{mg}$ cada 12 horas $^{22}$. Estudios desarrollados con dosis mensuales de penicilina $\mathrm{G}$ benzatina no han logrado demostrar un beneficio para evitar esta recurrencia en pacientes con factores de riesgo, aunque sí para aquellos pacientes sin esta condición ${ }^{23}$.

\section{Fascitis necrosante}

La reemergencia de infecciones graves por $S$. pyogenes, que en una fracción de los casos se presentan con cuadros cutáneos, pone de relieve su sospecha y diagnóstico diferencial adecuado. La manifestación cutánea más frecuente corresponde a un exantema generalizado y sólo $15 \%$ o menos se presenta con un cuadro de fascitis necrosante. Esta última condición se presenta con gran toxicidad sistémica e inicialmente con dolor de tejidos blandos, desproporcionado a la lesión cutánea visible. El cuadro es rápidamente progresivo y fulminante, con desarrollo de necrosis en el tejido subcutáneo y shock tóxico. La mitad de los casos se presenta en pacientes inmunocompetentes y el resto aparece asociado a pacientes con ciertos factores de riesgo tales como varicela, heridas o cortes en la piel de tipo quirúrgico o traumáticas, quemaduras, cirugía o parto vaginal ${ }^{2,24}$. Una placa de erisipela o celulitis estable no es parte de un cuadro de infección invasora por $S$. pyogenes. Algunas claves diagnósticas que permiten sospechar esta condición se resumen en la Tabla 6.

El manejo de estos cuadros es médico-quirúrgico, habitualmente en unidades de cuidados intensivos, incluyendo un esquema antimicrobiano combinado, inmunoglobulina endovenosa, terapia de sostén y un tratamiento quirúrgico según la circunstancias (aseos, debridamiento o amputación). La letalidad es elevada, especialmente si se acompaña de shock ${ }^{24}$. Las causas de la severidad

Tabla 6. Claves diagnósticas para sospechar una infección severa o cuadros de fascitis necrosante por Streptococcus pyogenes

- Presencia de shock o hipotensión.

- Cuadro sistémico o cutáneo fulminante y progresivo.

- Dolor cutáneo desproporcionado a la lesión cutánea visible.

- Alteraciones en las pruebas de coagulación o trombocitopenia.

- Factores de riesgo para fascitis necrosante: varicela, heridas traumáticas o quirúrgicas, quemaduras. de estos cuadros radican en las propiedades de virulencia de algunos integrantes de la especie $S$. pyogenes $^{24-26}$.

\section{Conclusiones}

Los cuadros de celulitis y erisipela son una causa importante de morbilidad y en ocasiones, de hospitalización. Los casos de erisipela tienen una manifestación clínica característica y se asocian generalmente a una causa estreptocóccica. Los factores de riesgo más importantes para su desarrollo están constituidos por linfedema de la extremidad afectada, la existencia de alteraciones cutáneas locales, la insuficiencia venosa, el edema de las extremidades o el sobrepeso. El diagnóstico puede ser apoyado por la presencia de anticuerpos específicos dirigidos contra estreptolisina $\mathrm{O}$, no presente en más de $40 \%$ de los casos y además positivo en infecciones por estreptococos de los grupos C o G. El tratamiento de elección de la erisipela es penicilina $G$ sódica o amoxicilina y su vía de administración está ligada a la severidad determinada clínicamente, reservando la administración parenteral para los casos más severos.

Los casos de celulitis tienen una manifestación clínica más indefinida y tienen un mayor espectro etiológico, predominando $S$. pyogenes y $S$. aureus en sus causas. La resistencia de $S$. aureus a penicilina obliga a plantear el tratamiento de esta condición con cloxacilina, cefalosporinas antiestafilocóccicas (cefazolina), clindamicina o preparados con inhibidores de $\beta$-lactamasas.

La emergencia de las infecciones severas e invasoras asociadas a $S$. pyogenes obliga a considerar esta posibilidad en casos de infecciones de piel y tejidos, fulminantes o asociadas a hipotensión y gran compromiso del estado general. En ellas, el tratamiento implica hospitalización y un adecuado enfoque médico-quirúrgico en una unidad de cuidados intermedios o intensivos.

\section{Resumen}

La celulitis y la erisipela son importantes causas de morbilidad e ingresos hospitalarios. La erisipela tiene una presentación clínica característica y se asocia típicamente a Streptococcus pyogenes y con menor frecuencia a estreptococos de los grupos B, C y G. Los factores de riesgo principales para desarrollar erisipela son linfedema y lesiones cutáneas locales. Una elevación de antistreptolisina $\mathrm{O}$ es indicativa de infección 
estreptocóccica, pero sólo se observa en $40 \%$ de los casos. El tratamiento de elección es penicilina $\mathrm{G}$ o amoxicilina y su duración y vía de administración están determinadas por la severidad del cuadro clínico. La celulitis es, en oposición, una infección cutánea y subcutánea sin límites precisos y tiene una etiología más amplia, incluyendo S. pyogenes y Staphylococcus aureus. La resistencia de este último a penicilina exige de un esquema terapéutico basado en cloxacilina, cefalosporina anti estafilocóccicas (p ej cefazolina), clindamicina o combinaciones con inhibidores de $\beta$-lactamasas. El surgimiento de infecciones invasoras por $S$. pyogenes obliga al reconocimiento precoz de esta condición; claves para su sospecha son la presencia de hipotensión, un deterioro general rápido, la necrosis tisular y existencia de un rash. Estas infecciones debieran ser manejadas con antimicrobianos parenterales, medidas de sostén general y un debridamiento quirúrgico agresivo.

\section{Bibliografía}

1.- Vaillant L. Diagnostic criteria for erysipelas. Ann Dermatol Venereol 2001; 128 (3 Pt2): 326-33.

2.- Bisno A L, Stevens D L. Streptococcal infections of skin and soft tissues. N Engl J Med 1996; 334: 240-5.

3.- Bernard P, Bedane C, Mounier M, Denis F, Catanzano G, Bonnetblanc J M. Streptococcal cause of erysipelas and cellulitis in adults. A microbiologic study using a direct immunofluorescence technique. Arch Dermatol 1989; 125: 779-82.

4.- Leppard B J, Seal D V, Colman G, Hallas G. The value of bacteriology and serology in the diagnosis of cellulitis and erysipelas. Br J Dermatol 1985; 112: 559-67.

5.- Bernard P, Toty L, Mounier M, Denis F, Bonnetblane J M. Early detection of streptococcal group antigens in skin samples by latex particle agglutination. Arch Dermatol 1987; 123: 468-70.

6.- Dupuy A, Benchikhi H, Roujeau J C et al. Risk factors for erysipelas of the leg (cellulitis): case-control study. Br Med J 1999; 318: 1591-4.

7.- Treiman G S, Copland S, Yellin A E, Lawrence P F, McNamara R M, Treiman R L J. Wound infections involving infrainguinal autogenous vein grafts: a current evaluation of factors determining successful graft preservation. Vasc Surg 2001; 33: 948-54.

8.- Woo P C, Lum P N, Wong S S, Cheng V C, Yuen K Y. Cellulitis complicating lymphoedema. Eur J Clin Microbiol Infect Dis 2000; 19: 294-7.

9.- Koutkia P, Mylonakis E, Boyce J. Cellulitis: evaluation of possible predisposing factors in hospitalized patients. Diagn Microbiol Infect Dis 1999; 34: 325-7.

10.- Thomas T A, Taylor S M, Crane M M et al. An analysis of limb-threatening lower extremity wound complications after 1090 consecutive coronary artery bypass procedures. Vasc Med 1999; 4: 83-8.

11.- Vinagre C, Cifuentes M, Valdivieso F, Ojeda A, Prado V. Emergencia de resistencia a macrólidos en Streptococcus pyogenes. Rev Méd Chile 1999; 127: 1447-52.
12.- Livermore D M, Williams J D. Beta-lactams: mode of action and mechanisms of bacterial resistance. En Lorian V (ed). Antibiotics in Laboratory Medicine. $4^{\text {th }}$ edition. Williams \& Wilkins, 1996, Baltimore, p 50278.

13.- Chambers H F. Penicillins. En: Mandell, Douglas and Bennett's Principles and Practice of Infectious Diseases. Mandell GL, Bennett JE, Dolin R (eds). $5^{\text {th }}$ edition. Churchill Livingstone, New York 2000; 26173.

14.- Svedhem A, Alestig K, Jertborn M. Phlebitis induced by parenteral treatment with flucloxacillin and cloxacillin: a double-blind study. Antimicrob Agents Chemother 1980; 18: 349-52.

15.- Steigbigel N H. Macrolides and Clindamycin. En: Mandell; Douglas and Bennett's Principles and Practice of Infectious Diseases. Mandell GL, Bennett JE, Dolin $\mathrm{R}$ (eds). ( $5^{\text {th }}$ edition). Churchill Livingstone New York 2000; 366-82

16.- Tassler H. Comparative efficacy and safety of oral fleroxacin and amoxicillin/clavulanate potassium in skin and soft tissue infections. Am J Med 1993; 94 (3A) $159 \mathrm{~S}-65 \mathrm{~S}$

17.- Amaya-Tapia G, Aguirre-Avalos G, Andrade-Villanueva $\mathrm{J}$ et al. Once-daily azithromycin in the treatment of adult skin and skin-structure infections. J Antimicrob Chemother 1993; 31 Suppl E: 129-35.

18.- Schupbach C W, Olovich K G, Dere W H. Efficacy of cefaclor AF in the treatment of skin and skin-structure infections. Clin Ther 1992; 14: 470-9.

19.- Parish L C, Jungkind D L. Systemic antimicrobial therapy in skin and skin structure infections: comparison of temafloxacin and ciprofloxacin. Am J Med 1991; 91 (6A): 115S-119S.

20.- Neldner K H. Double-blind randomized study of oral temafloxacin and cefadroxil in patients with mild to moderately severe bacterial skin infections. Am J Med 1991; 91(6A): 111S-114S.

21.- Dillon H C Jr. Treatment of staphylococcal skin infections: a comparison of cephalexin and dicloxacillin. J Am Acad Dermatol 1983; 8: 177-81.

22.- Kremer M, Zuckerman R, Avraham Z, Raz R. Longterm antimicrobial therapy in the prevention of recurrent soft-tissue infections. J Infect 1991; 22: 37 40 .

23.- Wang J H, Liu Y C, Cheng D L et al. Role of benzathine penicillin $G$ in prophylaxis for recurrent streptococcal cellulitis of the lower legs. Clin Infect Dis 1997; 25: 685-9.

24.- Fica A, Ulloa M T, Llanos C, Luzoro A, Porte L. Diversidad clínica de las infecciones invasivas por Streptococcus pyogenes y asociación con el superantígeno SpeA. Revista Hospital Clínico Universidad de Chile 2000; 11: 9-15.

25.- Ulloa M T, Giglio M S, Porte L et al. Detección de los genes de las exotoxinas pirogénicas de SpeA, SpeB y SpeC en cepas chilenas de Streptococcus pyogenes y su relación con la clínica. Rev Méd Chile 2000; 128: 27-34.

26.- Fernández J, Fica A, Caorsi B, Contreras J, Luppi M, Heitmann I. Síndrome de shock tóxico por Streptococcus $\beta$-hemolítico Grupo A. Estudio de la relación clonal entre un caso y sus contactos. Rev Méd Chile 1998; 126: 972-7.

Correspondencia a:

Alberto Fica Cubillos

E-mail: afica@ns.hospital.uchile.cl 